welche makroskopisch in Form strahliger und grubiger Einziehungen an der Innenfläche der Aorta sichtbar sind.

Dagegen läßt das Vorhandensein auch ausgedehnterer entzündlicher Prozesse in Adventitia und Media allein noch keinen Schluß auf die Ursache der Erkrankung zu.

\title{
XXVI.
}

\section{Über die fibrinösen Gerinnungen an der placentaren Fläche des Chorion.}

(Aus dem Parhologischen Institut der Universität Bern.)

Von

Dr. med. J. Biland.

Die ersten genauen Beschreibungen des Fibrins am Chorion frondosum rühren von Langhans und von Kölliker her. Kölliker unterscheidet 2 Formen des kompakten Fibrins. Die eine vergleicht er weichem Knochengewebe, in dem in einer hellen homogenen Zwischensubstanz zahlreiche kleine sternförmige Höhlungen sich finden, so daß das Ganze den Eindruck von Lamellen entkalkten Knochens macht. Mit Säuren behandelt kommen Kerne zum Vorschein and die Höhlungen erscheinen wie sternförmig anastomosierende Zellen. In der andern selteneren Form kommen an Stelle der Höhlungen anastomosierende Kanäle vor, mit Erweiterungen an den Knotenpunkten, hellem Inhalt, stellenweise mit Kern oder kernartigen Gebilden. Diese Kanäle sehen anastomosierenden Fadenpilzen täuschend ähnlich aus, und verdienen nach Kölliker den Namen von Saftkanälchen. Kölliker läßt beide Formen vom mütterlichen Blute aus entstehen.

Ausführlicher ist die Beschreibung von Langhans. Nach seiner Schilderung bildet das Fibrin, das von ihm mit dem Namen „kanalisiertes Fibrin“ belegt wurde, am Chorion eine kontinuierliche Lage, deren Dicke von wenigen hundertsteln Millimetern bis zu mehreren Millimetern wechselt, an beschränkten Stellen sogar eine Dicke von $1 \mathrm{~cm}$ erreichen kann. 
Die direkt auf dem Chorion gelegene Schicht des Fibrins ist am kompaktesten gebaut und zugleich am konstantesten, von der Mitte der Schwangerschaft an immer vorhanden. Es bildet eine homogene, weder deutlich fasrige, noch körnige, stark lichtbrechende, glänzende und dunkel konturierte Masse, die in Essigsäure aufquillt und blasser wird, in Karmin und Hämatoxylin sich etwas langsamer, dafür aber sehr intensiv und gleichmäßig färbt. In der stark glänzenden Grundsubstanz treten die Kanäle deutlich hervor. Diese sind von wechselnder Breite. Die breitern besitzen einen mehr runden Querschnitt, derjenige der feinern ist vielfach zackig, sternförmig. In der reifen Placenta wiegen die breitern Kanäle vor, sie verlaufen, durch senkrechte oder schräge Äste untereinander zusammenhängend, zur Oberfläche des Chorion parallel besonders in den obersten Schichten nach den intervillösen Räumen hin, so daß hier der Bau lamellös wird. In der Mitte der Schwangersehaft, im 5.-7. Monat, sind die queren oder schrägen Verbindungsäste zahlreicher und dichter und grenzen so mehr kugelige Massen gegeneinander ab (globuläre Form). Die Kanäle sind vielfach leer, oder enthalten kleine farblose Körnchen oder Kerne und Teile von solchen. Sie sind meist im Verlaufe der Kanäle eingeschaltet, seltener liegen sie an den Knotenpunkten. Auf dieser kompakten Form liegt die zweite Form, die lamellöse Form auf, deren Lamellen von der Fläche gesehen den gewöhnlichen retikulären Bau des Fibrins zeigen.

Was die Herkunft des Fibrins anlangt, so nimmt Langhans eine zweifache Genese an. Die kompakte Form leitet er von einer Lage großzelligen Gewebes auf der placentaren Fläche des Chorion $a b$, die er, wie er selbst nach wenigen Jahren nachwies, nichts anderes ist als die jetzt allgemein bekannte Zellschicht resp. faetales Ektoderm. Die lamellöse retikulare Form dagegen führt er auf das mütterliche Blut zurück. Die Grundsubstanz sollte aus dem Protoplasma der Zellen und einer geringen Menge Intercellulursubstanz (nach unsern jetzigen Kenntnissen „Fibrin“) durch Zusammenfließen entstehen, und die Kanäle führt er auf eine besondere Funktion der Kerne derselben zurück.

Die Untersuchungen von Langhans und Kölliker da- 
tieren aus einer Zeit, in welcher noch keine Finbettungs-Methoden und Mikrotome existierten, sowie auch die Färbetechnik noch sehr wenig entwickelt war. Namentlich die Einbettung in Celloidin hat unsere Kenntnisse über die Anatomie der Placenta außerordentlich gefördert. Seit der Einführung der Einbettungsmethoden sind wir besser daran. Wir können unsere jetzigen Kenntnisse über das Vorkommen von Fibrin in der Placenta der II. Hälfte der Schwangerschaft in folgendem zusammenfassen:

1. Es findet sich das Fibrin in fast kontinuierlicher Lage auf der placentaren Fläche des Chorion frondosum, wie das eben nach den ältern Untersuchụngen von Langhans und Kölliker beschrieben ist.

2. Es finden sich zwischen dan Zotten kleine Fibrininseln, die von Steffek und Eberhardt zuerst beschrieben wurden. Sie entstehen aus insulären Wucherungen der Zellschicht der Zotten, welche Langhans schon in seiner großen Arbeit unter dem Namen "großzellige Inseln" beschrieben hat.

3. Es findet sich Fibrin auf und in der Serotina; schon Langhans hat in der mehrfach erwähnten Arbeit darauf hingewiesen, daß sich hier ein dem kanalisierten Fibrin ähnliches Gewebe findet.

Dann hat namentlich Nitabuch, welche unter der Leitung von Langhans arbeitete, nachgewiesen, daß hier neben dem oberflächlichen direkt an den intervillösen Raum angrenzenden Fibrinstreifen noch ein zweiter, in der Dicke der Serotina gelegner sich findet (im VI. Monat der Schwangerschaft), welcher als Grenze zwischen dem tiefen mütterlichen Gewebe der Serotina und den obern ektodermalen Schichten derselben anzusehen ist.

Das Auftreten dieses Fibrins wird verständlich auf Grund einer Auffassung von Langhans, die in der Arbeit von Merttens niedergelegt ist (S. 51).

Es ist hier zum erstenmal klar ausgesprochen, daß dem Syncytium ein gerinnungshemmender Einfluß, der Zellschicht dagegen ein gerinnungsbefördernder Einfluß zukommt. Ferner ist die Tatsache hervorgehoben, daß die Existenz des Syncytium gebunden ist an das Vorhandensein eines 
foetalen Kapillarsystems, in den oberflächlichsten Schichten des Bindegewebes des Chorion und der Zotten. In den früheren Stadien ist die ganze Oberfläche des Chorion, sowie alle Zotten mit einem solchen Kapillarsystem versehen und dementsprechend findet sich anch hier überall ein kontinuierlieher Überzug von Syncytium.

"Wird infolge der Größenzunahme des Eies unter der Dehnung der Reflexa der Stoffaustausch zwischen Mutter und Frucht am Chorion laeve erschwert, so gehen beide Elemente zugrunde, Syncytium (Merttens hat dasselbe nach seiner Auffassung als Uterusepithel bezeichnet) und Blutkapillaren des Chorion laeve. Dasselbe tritt beim Chorion frondosum gegen die Mitte der Schwangerschaft ein, wenn die mächtige Entwicklung der Zotten, das Chorion von den zuführenden mütterlichen Blutgefäßen abdrängt. Man könnte also den Schwund des Uterusepithels als eine funktionelle Atrophie auffassen. Ebenso schwindet es an den Ektoderminseln und auf dem serotinalen Ektoderm."

Infolge dessen kommt nunmehr das mütterliche Blut an diesen Stellen in direkte Berührung mit den Elementen der Zellschicht und überall tritt jetzt Gerinnung ein.

Wie man hieraus ersieht, sind diese Ideen für die eigentliche Umwandlung, welche die Placenta in der zweiten Hälfte der Schwangerschaft erleidet, von der größten Bedeutung. Ich habe daher zunächst durch weitere Untersuchung festzustellen gesucht, ob die von Langhans behauptete Zusammengehörigkeit von Syncytium und foetalen Kapillaren in dem gegenüberliegenden Chorionbindegewebe, besteht. Ich habe daraufhin eine Anzahl von Abortiv-Placenten und einige reife Placenten, im ganzen 14, untersucht; von diesen Placenten entsprachen die jüngste Placenta dem 3. Schwangerschaftsmonat, drei weitere dem 4. Monat, eine dem 5., drei dem 6., zwei dem 8. Monat. Zwei Placenten waren nahezu ausgetragen (2-3 Wochen vor dem normalen Ende der Schwangerschaft), zwei weitere waren ausgetragen. Von diesen 14 Placenten bekam ich 6 Placenten frisch durch die Güte von Professor Müller aus der kantonalen Gebäranstalt in Bern. Von den Abortiv-Placenten fehlen mir meist klinische Notizen. 
Aus jeder einzelnen Placenta wurden Scheiben herausgeschnitten, die von einem Placentarrande durch die Mitte der Placenta zum andern reichten. Diese Seheiben wurden in kleinere Stücke zerlegt, die nach der Serotina gelegene Hälfte der Zoltenmassen samt der Serotina abgeschnitten und entfernt. Mit Celloidin durchtränkt, wurden diese Stüeke mit dem Mikrotom in Schnitte von 0,01-0,015 mm Dicke zerlegt. Zur Färbung bediente ich mich vorzugsweise des Hämatoxylin und Eosins und zwar so, daß ich die Schnitte im Eosin überfärbte und sie nachher während Stunden im Alkohol sich differenzieren ließ. Ferner wendete ich auch die Färbungsmethode nach van Gieson an. Die Schnitte wurden in Origanumöl betrachtet. Dabei achtete ich auf die Beschaffenheit des Syncytium, der L angh a nsschen Zellschicht and des Fibrin am Chorion und wie bereits erwähnt, auf den Kapillarenreichtum des Chorionbindegewebes bei vorhandenem Syncytium und bei eingetrener Fibrinbildung. Besonders lehrreich waren die Schnitte der Placenten, bei denen am nämlichen Schnitt das Chorion abwechselnd streckenweise ein Syncytium, streckenweise eine Fibrinschicht aufwies.

Ich schildere zuerst in Kürze das histologische Bild der einzelnen Placenten.

Placenta I. Die jüngste der 14 Placenten stammt aus dem dritten Schwangerschaftsmonat (Fötns $16 \mathrm{~cm}$ lang).

Das Syncytium ist fast auf der ganzen Ausdehnung des Chorion erhalten. Es besteht aus nebeneinander, aber dicht gelagerten, der Oberfläche des Chorion parallel verlaufenden, dunkelgefärbten länglieben Kernen, die häufig große Chromatinkörner aufweisen und in einem gemeinsamen fein granulierten Protoplasmasaum liegen, und zwar in dessen tiefern Schichten. Die unter dem Syncytium sich befindende Zellschicht ist einschichtig; die einzelnen deutlich voneinander getrennten Zellen länglich bis quadratisch; der Zellleib ist hell, der Kern bläschenförmig blaß, meist oval, zuweilen polyedrisch. Im Kern ist ein feines, gleichmäßig verteiltes Chromatingerüst und ein durch das Eosin rot gefärbter Nucleolus sichtbar. Letzterer tritt bei der Färbungsmethode nach van Gieson besonders deutlich hervor. Jede einzelne Zelle springt ins Chorionbindegewebe hinein vor. Die Grenze zwischen Zellschicht und Chorion wird so von welligem Verlaufe. Die Zellen sind stellenweise auseinandergerückt. In den Zwischenräumen scheint das Syncytium direkt das Chorionbindegewebe zu berühren, nur zuweilen schiebt sich zwischen beide noch eine schmale helle, der Zellschicht angehörende Platte eir.

An den seltenen und wenig ausgedehnten Stellen, an denen Fibrinbildung eingetreten und das Syncytium geschwunden ist, weist die Zellschicht eine mehrschichtige (drei- bis vierfache) Anordnung ihrer Zellen auf. Diese letzeren sind eher klein, weisen einen großen bläschenförmigen, blassen, mit einem eosinrotgefürbten Nucleolus versehenen Kern auf; die Verteilung der Chromatinkörner ist häufig unregelmäßig, in dem die- 
selben oft der Kernmembran zu liegen. Die Zellen springen gruppenweise "nischenartig" ins Chorionbindegewebe hinein vor und bilden eine ununterbrochene Zelllage, die das Chorion vom Fibrin trennt. Zwischen den Zellen der Zellschicht finden sich ziemlich zahlreiche Leukocyten vor. Das Fibrin ist von geringer Dicke, mehr kleinschollig-balkig und endet über der Zellschicht in ebener Fläche, ohne Ausläufer zwischen die Zellen derselben zu versenden, zeigt somit das Bild einer Fibrinauflagerung.

Im Bindegewebe des Chorion sind zahlreiche Kapillaren, durchschnittlich eine pro Gesichtsfeld, ${ }^{1}$ ) direkt an der Oberfläche, von denen einzelne eine Adventitia zeigen.

\section{Placenta aus dem 4. Monat.}

Placenta II. Infolge eines künstlichen Abortus im Februar 1901 dem pathologischen Institut zugestellt.

Das Syncytium mit seiner ununterbrochenen Reihe dichtstehender länglicher dunkler Kerne bedeckt das Chorion auf dem größten Teile seiner Oberfläche. Unter ihm sind die Zellen der Zellschicht, die sich durch ihre Größe, ibren hellen Zellieib, ihren blassen ovalen Kern kennzeiehnen. Auf 4 bis 5 Kerne des Syncytium kommt eine Zelle der Zellschicht. Auch hier springt jede einzelne Zelle ins Chorionbindegewebe hinein vor. Nur an wenigen Stellen findet sich Fibrin, mit Vorliebe da, wo Zottendurchschnitte dicht an das Chorion zu liegen kommen. Beim. Übergang in diese Stellen verliert die Zellschicht ihre einreibige Anordnung, sie wird mehrschichtig, bildet immer noch. rom Syncytium bedeckt, Zellhaufen, die nischenartig ins Chorionbindegewebe hinein vorspringen. Meist liegen 2 bis 4 Zelllagen übereinander, in einem Zellhaufen finden sich 8-12 Zellen vor. Da wo das Syncytium über den Zellhaufen aufhört, findet sich eine Fibrinschicht von mäBiger Dicke vor, die in den obersten Schichten teils streifenförmig lamellös und weitspaltig ist, teils aus kleinsehollig-balkigem oder aus einem Netz von Fibrinfasern besteht, in dessen Maschen rote und weiße Blutkörper liegen. Gegen die Zellschicht hin ist das Fibrin kompakt, bildet breite stark lichtbrechende Balken, häufig zeigt es auch die globulöse Form der Anordnung. In den Zwischenräumen zwischen den Balken liegen kleine runde, oft buchtige, dunkle Kerne und Kerne mehrkömiger Leukocyten. Von dem direkt über der Zellschicht liegenden Balken gehen Ausläufer in Form von gröbern und feinern Fasern zwischen die Zellen der Zellschicht hinein, so daß die letzzteren vou diesen Zügen allseitig begrenzt werden. Diese Zellen sind, was Kern nnd Zellleib anbelangt, meist bedeutend größer, als die näher dem Chorion anliegenden Zellen. Die Verteilung der Chromatinkörner in den Kernen jenex Zellen ist zum Teil noch eine gleichmäßige, zum Teil sind die Chromatinkörner mehr periphär angeordnet. Die Kerne der

1) Das .im Sinne eines Flächenmaßes gebrauchte Gesichtsfeld“ kehrt im inachstehenden häufiger wieder. Ich verstehe darunter das Gesichtsfeld von Leitz 1/192 Immersion, Okk. I. 
am weitesten gegen die Fibrinschicht hin gelegenen Zellen haben ihre Färbbarkeit verloren, bilden Schollen, in denen der Kern nur noch als Schatten infolge der verschiedenen Brechung des Lichtes hervortritt. Solche Schollen finden sich stellenweise auch zwischen den noch gut erhaltenen Zellen der Zellschicht. Im Chorionbindegewebe sind an der Oberflïche desselben Kapillaren in wäßiger Menge (eine auf zwei Gesichtsfelder) vorhanden, und zeigen zuweilen eine Adventitia, Da wo eine Fibrinschicht vorhanden ist, zeigt das Chorion keine Gefäße.

PIacenta III aus dem 4. Monate. Abortus vom 26. X. 01.

Das Syncytium ist auf dem gröBten Teil der Oberfäche des Chorion erhalten, die Kerne zeigen die bekannten Merkmale, liegen stellenweise dicht beisammen, stellenweise ziemlich weit auseinander und sind teils sehr lang. Darunter ist die einreihige Linie der Zellen der Zellschicht. Da wo das Syncytium fehlt, liegt eine mehr oder weniger breite Fibrinschicht der hier mehrschichtigen Zellschicht auf. Das Fibrin ist dem Chorion zunächst kompakt, nach oben zu globulös und lamellös, weist untereinander kommunizierende, dem Chorion parallel verlaufende Kanäle auf, in denen dunkelgefärbte, ovale, oft verästelte Kerne, gekörnte Schollen, zuwreilen rote Blutkörper und auffallend viele mehrkörnige weiße Blutkörper vorkommen. Diese letzern finden sich auch zahlreich zwischen den Zellen der Zellschicht vor. Die Zellen der Zellsehicht sind durchschnittlich klein, nur um ein weniges größer als ein weißes Blutkörperchen und liegen meist drei bis vier übereinander. Sie bilden stellenweise Zellhaufen, sind meist polyedrisch, besitzen einen hellen Zellleib und einen in der Form vom Ovalen bis ganz Unregelmäßigen schwankenden Kern. Nur an wenigen Stellen scheint die Zellschicht zu fehlen und das Fibrin direkt auf dem Bindegewebe des Chorion zu liegen. An diesen Stellen findet sich häufig ïber dem Fibrin noch ein Syneytium vor. Im Chorion sind die Kapillaren sehr spärlich; da wo eine Fibrinschicht vorhanden ist, fehlen sie ganz. Wie im Fibrin und in der Zellschicht trifft man anch im Bindegewebe des Chorion auf starke Leukocyteninfiltration.

Placenta IV, aus dem 4. Monat, dünn; Foetus $19,5 \mathrm{~cm}$ lang.

Das Syncytium ist auf dem Chorion nur stellenweise vorhanden und zeigt das gewöhnliche Verhalten. Unter ihm sind, einreihig angeordnet, dje mit einem bläschenförmigen Kern und einem Kernkörperchen versehenen Zellen der Zellschicht. Meistens fehlt das Syncytium. Eine meist ziemlich dicke Fibrinschicht liegt der hier mehrschichtigen Zellschicht auf. Nur selten scheint das Fibrin die Oberfläche des Chorion direkt zu berühren. Die Zellen der Zellschicht, meist zu dreien oder vier, zuweilen aber auch viel zahlreicher, bis zehn bis fünfzehn übereinander, bilden kontinuierliche Reihen, oder ebenso häufig Zellhaufen, die konvex ins Chorionbindegewebe hinein vorspringen. Sie sind im ganzen klein, haben einen hellen zuweilen fein granulierten Zellleib, und einen gut erhaltenen bläschenförmigen blassen, mit einem gleichmäßig verteilten Chromatingerüst und einem Nucleolus versehenen Kern. Da wo sie weniger dicht 
gelagert and deshalb deutlicher voneinander abzugrenzen sind, sind sie quadratisch oder zylindrisch. Vereinzelte ins Chorion konvex vorspringende Zellhaufen weisen große, längsovale Elemente auf, oft vier bis fünf übereinander, die jedes von Fibrinzügen eingeschlossen ist. Das Fibrin grenzt mit einem schmalen, homogenen, stark lichtbrechenden Balken an die Zellschicht an, von dem sich stellenweise Fibrinzüge ablösen, in die Zellschicht eindringen, die Zellhaufen in kleinere Gruppen teilen und zuweilen die Zellen allseitig umfassen. In den meist zur Dicke der Fibrinbalken ziemlich breiten Zwischenräumen sind spärliche, helle, bläschenförmige und zahlreiche kleine, dunkle, längliche, oft zackige Kerne sichtbar. Auf diese homogenen Fibrinbalken folgen gegen den intervillösen Raum hin Schollen und Klumpen von Fibrin, die durch mehr oder weniger schmale, kleine, dunkle Kerne aufweisende Gänge voneinander getrennt sind. Den Uhergang in den Inhalt des intervellösen Raums bildet eine homogene feinkörnige gelbliche Zone. Da wo die mehrschichtige Zellschicht eine mehr gleichmäBige Breite hat, werden die einzelnen Zellagen häufig durch dem Chorion parallel verlaufende Fibrinfasern voneinander geschieden. Diese Fasern, die durch schräg verlaufende oder senkrechte Zweige untereinander zusammenhängen, liegen gegen das Chorion hin dichter und schließen öfters nekrotisierende Zellen der Zellschicht zwischen sich ein.

Die Gefäße im Chorion sind selten. Doch zeigt sich, wie schon bei den vorhergehenden Placenten, nur ansgesprochener, ein später oft wiederzufindender Unterschied im Vorkommen derselben. Da wo Fibrinbildung eingetreten ist, sind sie äußerst spärlich, ja fehlen auf langen Strecken vollständig. Auf 75 Gesichtsfelder fand sich eine einzige Kapillare vor, währenddem an den Stellen, an denen die Fibrimbildung noch ansgeblieben ist und ein Syncytium die einreihige Zellschicht bekleidet, die Kapillaren viel häufiger vorkommen. Auf den 12 Gesichtsfeldern fanden sich $17 \mathrm{Ka}$ pillaren.

\section{Placenta $V$, aus dem 5. Monate.}

Diese Placenta stammt von einer am 2. VI. 03 in extremis in die kantonale Gebäranstalt gebrachten Frau her. Die Geburt wurde durch Einlegen eines Ballon beschleunigt. Der Tod der Frau, infolge chronischer Nephritis, erfolgte einige Stunden post part.

Das Chorion weist auf fast der ganzen Ausdehnung eine ziemlich dicke Febrinschicht auf, die stellenweise mehr oder weniger gut erhaltene Zotten einsehlieRt und überall dureh eine meirschichtige Zellsehicht von der Oberfläche des Chorion getrennt ist. Nur dicht oberbalb der Zellschicht und zuweilen inmitten des Fibrins, da wo die Fibrinschicht dünn ist, ist das Fibrin kompakt. Im übrigen ist es kleinschollig, klumpig. Meist grenzt es geradlinig an die Zellschicht, oder es springt in Form von zackigen Ausläufern zwischen die zu oberst gelegenen Zellen der Zellschicht vor und schließt dieselben von allen Seiten ein. In den zwischen den Fibrinbalken und Klumpen vorhandenen schmalen Spalten sind sehr spärlich dunkle Kerne sichtbar. Die Zellschicht ist mehrschichtig; die 
Zellen liegen zu vier bis acht übereinander, sind dichtgedrängt, zeigen einen hellen oft fein granulierten Zelleib und im Zentrum einen rundovalen, einen exzentrisch gelagerten Nucleolus enthaltenden Kern. Die Grenze zwischen den Zellen bildet eine feine, oft lichtbrechendes Vermögen aufweisende Linie. Die Größe der einzelnen Zellen schwankt. Die kleinsten sind durchschnittlich mehr chorionwärts gelegen. Der Größe der Zelle geht meist die des Kerns parallel. Stellenweise finden sich große Vakuolen inmitten der Zellschicht vor. Wie schon erwähnt, liegen die dem Fibrin zunächst gelegenen Zellen in den Maschen eines Fibrinnetzes und zeigen dann meist Veränderungeu in ihrer Struktur. Der Zellleib ist oft grob gekörnt, der Kern kleiner, oft ganz geschwunden, so daß im Fibrin nur noch eine grobkörnige Protoplasmamasse enthalten ist. In der Nähe von Zottendurehschnitten ist die Fibrinschicht anf einen schmalen Streifen reduziert und auf ihm ein syncytialer Überzug vorhanden. Im Chorion sind keine Kapillaren sichtbar. Dagegen durchwegs zwischen den Bindegewebsbündeln kleine Haufen gelbbraunen Pigments.

Placenten aus dem 6. Monat, Placenta VI.

Das Syncytium ist am Chorion nur stellenweise vorhanden, so daB auf dem nämlichen $3 \mathrm{~cm}$ langen Schnitte Syncytium und Fibrinschicht 3 bis 4 Male miteinander abwechseln. Es zeigt, sowie die unter ihm gelegene Zellschicht normales Verhalten. Die dem Chorion aufliegende Fibrinschicht ist verschieden dick und scheint stellenweise das Chorion direkt zu berühren. Sie ist teils balkig, teils klumpig; teils weist sie einen netzartigen, einer diphtheritischen Membran ähnlichen Ban auf. Bei den beiden erstgenannten Formen des Fibrins kommen Kanäle vor, die unregelmäßig gestaltet, untereinander netzartig zusammenhängen und gegen den intervillosen Raum hin weiter und verzweigter sind. Gegen den intervillösen Raum hin fasern sich die Fibrinlamellen oder Balken in ein Netz von Fibrinfäden auf, das rote und weiBe Blutkörper einschließt und sich allmählich in Inhalt des Intervillösenraumes verliert.

Die Zellen der Zellschicht sind zu mehreren Schichten angeordnet, die teils kontinuierlich sind, teils Zellgruppen oder Zellhaufen bilden, die unter Bildung von Nischen ins Chorionbindegewebe hinein vorspringen. Die einzelnen Zellen sind in der dem Chorion aufsitzenden Schicht quadratisch, in den oberen Schichten rundlich, oval, polyedrisch. Die Zellgrenze ist als feine Linie erkenntlich; der Zellleib hell, der Kern rund, oval, oft unregelmäßig, meist groß, 3-5́mal, ja einzelne Elemente bis 10 mal den Durchmesser eines roten Blutkörpers übertreffend; im Kern ein deutliches Chronatingerüst and ein bis mehrere Kernkörperchen. Im allgemeinen scheinen die Zellen gegen den Intervillösenraum hin größer zu werden. Da wo die Zellschicht Zellhaufen bildet, liegen die Zellen oft zu 10 bis 20 übereinander. Währenddem sie gegen das Chorion dicht aneinander liegen, schieben sich in den mittlern Lagen Balken von Fibrin zwischen die Zellen ein, verlaufen meist parallel zur Oberfläche des Chorion und gehen zuweilen, miteinander zusammenfließend, in große Komplexe 
von Zellgröße über. Die angrenzenden Zellen weisen häufig Körnelung ihres Zellleibs auf. Gegen den Intervillösenraum hin wird der Zellverband zwischen den Zellen der Zellschicht ein lockerer, oft findet man losgelöste zu kleinern Gruppen beisammenliegende, oft vereinzelte Zellen mitten in der kompakten Fibrinschicht. Die Kernfärbung dieser Zellen ist schlecht, häufig ist nur noch ein gekörnter Zellleib als Überrest der Zelle vorhanden. Im Chorion sind die GefäßBe sehr spärlich; da wo Fibrinbildung vorkommt, $o b$ die Zellschicht darunter vorhanden sei oder nicht, fehlen die Kapillaren durchwegs. Dagegen an den wenigen Stellen, die ein Syncytium aufweisen, finden sich an der Oberfläche des Chorion kleine Kapillaren.

Placenta VII, aus dem 6. Monate.

Auf einem Teil der Blöcke zeigt das Chorion in fast ganzer Ausdehnung ein Syncytium, auf dem andern Teil war das Chorion größtenteils von einer Fibrinschicht bedeckt.

a) Strecken mit Syncytium. Das Syneytium besteht zumeist aus länglichen, schmalen, dunkelgefärbten Kernen, die dem Chorion parallel liegen, zuweilen auch schräg zur Oberfläche des Chorion gestellt sind. Die Kerne liegen stellenweise sehr dicht und zeigen daselbst zuweilen eine mehr ovale Form. Doch sind die Zellen der Zellschicht an dem hellen Zellleib, dem viel größern ovalen blassen Kern mit Kernkörperchen, stets dentlich erkenntlich. Die Zellen sind oval, quadratisch bis polyedrisch, springen einzeln ins Chorionbindegewebe hinein vor, liegen zuweilen dichter, meist aber in kleinen Abständen auseinander. Das Syncytium grenzt im allgemeinen unmittelbar an den Inhalt des intervillösen Raums an. Mancherorts schiebt sich aber ein schmaler, homogener, glänzender, gelber Saum ein, der sich aus zusammengeflossenen roten Blutkörpern aufzubauen scheint.

b) Beim Übergang in die Stellen, an denen Fibrinbildung eingetreten ist, sieht man das Syncytium sich noch eine kleine Strecke auf das Fibrin fortsetzen. Die Kerne schwinden aber bald und nur der schmale Protoplasmasaum ist auf eine kurze Strecke noch erkenntlich, bis er in dem das hier das benachbarte Syncytium überragenden Fibrin verschwindet. Die Fibrinschicht wechselt in ihrer Dicke, stellenweise ist sie sehr dünn, und kommt 2 bis $3 \mathrm{mal}$ der Breite des Syncytium gleich; stellenweise wiederum ist sie sehr dick, und übertrifft die Dicke des Chorion oft mehrmals. Da wo sie dünn ist, besteht sie in toto aus kompaktem Fibrin, und enthält feine, unregelmäßige, verästelte Kanäle von wechselndem Durchmesser, in denen stellenweise dunkle unregelmäBig gestaltete, mit Ausläufern versehene Kerne sichtbar sind. Da wo die Fibrinschicht mächtiger ist, verliert sie meist ihre kompakte Struktur und besteht aus mehr locker gefügten Balken kleinscholligen Fibrins, oft weist es mehr netzartigen Bau auf. In den Maschenräumen des zuletzt geschilderten Fibrins und in den von den Balken begrenzten unregelmäßig geformten Lücken und Kanälen, die gegen das Chorion zu schmäler und feiner werden, sind klumpige und körnige Fibrinmassen, sowie viele unregelmäßig gestaltete dunkelgefärbte 
Kerne enthalten. Ganz allmählich, unter Auffaserung der Balken, Feiner-undFeinerwerden der Fibrinfäden und Einschließen von weißen und roten Blutkörpern geht die Fibrinschicht in den Inhalt des intervillösen Raumes über. Meist scheint das Fibrin die Chorionoberfläche direkt zu berïhren, streckenweise ist es aber durch eine mehrschichtige, 2 bis 3 Zelllagen übereinander zählende Zellschicht von derselben getrennt. Die Zellen bilden meist Haufen, die sowohl in das Bindegewebe des Chorion, als in das kompakte Fibrinkonvex vorspringen. Sie liegen dicht beisammen und sind zumeist gut erhalten und durchschnittlich klein. Nach oben zu grenzen die Zellhaufen scharf $a b$, ohne aus ihnen losgelöste and ins Fibrin eingesprengte Zellen zu zeigen. Die an die Zellschicht angrenzende und aus kompaktera Fibrin bestehende Fibrinlage sendet stellenweise feine, oft zu Büscheln zusammenliegende Fasern zwischen die Zellhaufen hinein, und schließen diese von den Seiten ein, oder die Fasern verlaufen dem Chorion parallel und trennen die einzelnen Schichten der Zellschicht voneinander, wodurch diese einen etagenähnlichen Aufbau gewinnt. Wie schon erwähnt, sind die Zellen meist gut erhalten, bisweilen hat aber der Kern an Färbbarkeit eingebüBt, oder der Kern ist ganz geschwunden und an Stelle der Zelle noch Protoplasmaschollen vorhanden. An verschiedenen Stellen sind zu Haufen beisammenliegende Syncytiumkerne zwischen der an diesen Strecken ziemlich dicken Fibrinschicht und der eingedrückten Zellschicht eingeklemmt.

Da wo das Syncytium vorhanden ist, finden sich Kapillaren an der Oberfläche des Chorionbindegewebes vor; sie sind meist unregelmäßig verteilt, in den einen Gesichtsfeldern nur eine Kapillare, in anderen wiederum 4-5, einzelne zeigen eine Adventitia. Auf den Strecken, auf denen das Chorion von einer Sehicht Fibrin bedeckt ist, kommen, da wo das Fibrin dünn ist, noch ganz vereinzelte Kapillaren vor, da wo es dick ist, fehlen sie ganz.

\section{Placenta VIII, aus dem 6. Monat.}

Das Syncytium, das auf kurze Strecken neben den teils flächenhaften, teils knospenförmigen Auflagerungen von Fibrin am Chorion vorkommt, zeigt das gewöhnliche histologische Bild. Ebenso die unter ihm gelegene Zellschicht. Das Fibrin bildet eine ziemlich dicke Schicht (von der Breite des Chorion), wird am Rande meistens auf eine kleine Strecke noch vom Syncytium überzogen, das sich dann in einen Fibrinbalken fortzusetzen scheint. Es schlieBt zahlreiche Zotten ein und grenzt gegen den intervillösen Raum scharf ab. Das Fibrin besteht aus breiten homogenen Balken, weiter gegen den intervillösen Raum hin ist es bald balkigkleinschollig, klumpig, oder wieder von netzförmigem Bau. Es weist zahlreiche Gänge auf von unregelmäßiger Form und unregelmäßigem, zackigem Querschnitt, die gegen das Chorion hin einen demselben parallel gerichteten Verlauf nehmen, währenddem sie nach oben zu keine bestimmte Richtung besitzen. In diesen Kanälen finden sich kleine rundliche bis unregelmäßige, buchtige dunkle Kerne vor. Das Fibrin berührt fast nirgends direkt die Oberfläche das Chorion, sondern ist durch eine 2 bis 4 schichtige 
Zellschicht von demselben getrennt. Die Zellen der Zellschicht sind klein, liegen teils in Gruppen von dreien, oder haufenweise beisammen und springen dann ins Chorionbindegewebe hinein vor. Zwischen den Zellen sind stellenweise homogene Fibrinmassen oder Balken von Fibrin, die die einzelnen Zellen von einander abgrenzen. Am Rande einer Fibrinplatte, von derselben bedeckt, setzt sich das Syncytium eine Strecke weit fort. Unter ihm liegt eine einschichtige normale Zellschicht. Dann verliert sich das Syncytium, scheint in einen Fibrinbalken überzugehen und das Fibrin berührt jetzt erst direkt die Zellschicht. Es hat den Anschein, als ob an letzterer Stelle die Fibrinbildung begonnen und sich von da pilzförmig, in Form einer Platte, über die ausstoßenden von einem Syncytium bedeckten Partien des Chorion ausgedehnt hätte. Im Chorion kommen die Kapillaren nur da vor, wo ein Syncytium besteht, sind aber spärlich, so daß auf 4-5 Gesichtsfelder nur eine Kapillare kommt. Im übrigen fehlen die Kapillaren ganz. Auf einem der Schnitte ist die Abgangsstelle einer Zotte getroffen. An der Basis der Zotte, da wo diese die Fibrinschicht des Chorion durchbricht, kommen einige wenige Kapillaren vor; außerhalb der Fibrinschicht sind die Kapillaren zahlreich unter dem Zotten-Syncytium vorhanden.

\section{Placenten aus dem 8. Monat.}

Placenta IX wurde bei einem Exitus infolge Pyelonephritis 4 Stunden nach dem Tode gewonnen.

Das Syncytium ist am Chorion nur stellenweise vorhanden, oft nur an den Abgangsstellen von Zotten, und bildet meist eine kontinuierliche Reihe von Kernen. Unter ihm liegen in einschichtiger Reihe die Zellen der Zellschicht. Beide, Syncytium sowohl als Zellschicht, weisen die gewöhnten Merkmale auf. An einigen Stellen, über der mehrschichtig gewordenen Zellschicht, zwischen deren Zellen sich bereits homogene Zwischensubstanz vortäuschendes Fibrin eingeschoben hat, finden sich noch Uberreste des Syncytium vor: eine in einem Protoplasmasaum liegende, mehr oder weniger lange Kette langer, schmaler, dunkler Kerne.

Das Fibrin bildet eine ziemlich gleichmäBige Lage, die an Dicke ungefähr der Breite des Chorion gleichkommt. Sie ist vom Chorion, nur wenige Stellen ausgenommen, durch die Zellschicht getrennt. Diese letatere ist teils 2-3 schichtig; oft aber viel mächtiger entwickelt, so daß 10 bis 15 Zellen übereinander zu liegen kommen. Die einzelnen Zellen sind ziemlich groß, liegen dem Chorion zu dichter und sind daselbst, besonders was den Zellleib anbelangt, etwas kleiner als die übrigen Zellen. Die Zellgrenze ist als eine feine lichtbrechende Linie sichtbar. Meistens sind die Zellen zu Haufen angeordnet, springen ins Chorionbindegewebe hinein vor, oder sie sind als Zellgruppen oder als vereinzelte Zellen in das Fibrin eingesprengt, so daß letzteres an diesen Stellen direkt das Chorion zu berühren scheint. Häufig finden sich zwischen den zu Zellhaufen angeordneten Zellen Balken aus einer homogenen Substanz, die die Zellen teilweise, oft allseitig begrenzen und direkt mit dem kompakten Fibrin 
zusammenhängen, das die Zellhaufen gegen den intervillösen Raum hin bedeckt. Die Zellen selbst sind zum Teil gut erhalten, zum Teil zeigen sie einen gekörnten Zellleib and einen schlecht gefärbten Kern. Gegen den intervillösen Raam hin bleibt bei den vom Fibrin umschlossenen Zeilen die Kernfärbung aus, sie stellen nur noch eosinnot gefärbte Protoplasmaschollen dar. Die Zellen, die vereinzelt im Fibrin eingesprengt liegen, füllen meist den ihnen in demselben eingeräumten Raum nicht vollständig aus; sondern zwischen Zelle und Fibrin besteht ein Spaltraum. Je weiter diese Zellen vom Chorion abliegen, um so größer und unregelmäßiger werden die Spalten; die Spalten der verschiedenen benachbarten Zellen treten untereinander in Verbindung, und bilden so ein ganz unregelmäßiges verzweigtes Gangsystem, das gegen den intervillösen Raum hin in zur Chorionoberfläche parallel gerichtete Kanäle übergeht. Das Fibrin ist bis hierher kompakt und geht dann noch oben in balkig-kleinscholliges Fibrin über, dessen Kanäle wieder verzweigter und größer sind, durch senkrechte und schräge Seitenäste untereinander in Verbindung treten; schließlich löst sich das Fibrin in ein Netz von Fibrinfäden auf, das einer diphtheritischen Membran ähnlich sieht, und das immer feiner werdend, unter Einschließen von Leukocyten, Lymphocyten und roten Blutkörpern in den Inhalt des intervillösen Raumes übergeht. Da wo die Zellschicht mächtig ist, ist die Fibrinschicht sebr dünn und besteht aus Bändern, die sich aus feinen Fasern zusammensetzen und ebenfalls unter Einschließen von roten und weißen Blutkörpern in den Inhalt des intervillösen Raumes übergehen. In den Kanälen des Fibrin sind nur spärliche Kerne sichtbar, sie liegen meist gegen den intervillösen Raum hin.

An einigen Stellen kommen mitten in der Zellschicht GefäBe vor, ziemlich zahlreiche, quer und schräg getroffene Kapillaren, deren Lumen von roten Blutkörperchen erfüllt ist. Die wenige schmale längsverlaufende Endothelkerne aufweisende Gefäßwand stößt direkt an die Zellen der Zellschicht an und ist deutlich von denselben zu trennen.

Da ich leider unterließ, Serienschnitte anzulegen, kann ich nicht entscheiden, ob diese Kapillaren mit den mütterlichen oder foetalen Gefäßen in Zusammenhang waren.

Was den Kapillarreichtum des Chorionbindegewebes anbelangt, so ist derselbe, da wo ein Syncytium und eine normale einschichtige Zellschicht das Chorion bekleiden, sehr groß. Auf jedes Gesichtsfeld fallen $2-3 \mathrm{Ka}-$ pillaren, auf 32 Gesichtsfelder traf es 58 Kapillaren. Da wo die Zellschicht noch von einem Syncytium bedeckt ist, aber mehrschichtige Anordnung und Fibrinbildung zwischen den Zellen erkennen läßt, sind die Kapillaren spärlicher, 1 Kapillare pro 1-2 Gesichtsfelder. Da wo eine Fibrinschicht das Chorion bedeckt, sind die Kapillaren selten, 3 Kapillaren auf $36 \mathrm{Ge}$ sichtsfelder.

PIacenta $X$, aus dem 8. Monate, von einer Frühgeburt bei engem Becken herrïhrend.

Das Syncytium fehlt am Chorion fast durchwegs, außer an den Stellen, an denen ein Zottenstamm vom Chorion abgeht. Das Fibrin des Chorion 
reicht daselbst bis direkt an den Zottenstamm heran, und das Syncytium der Zotten setzt sich auf dieses Fibrin noch fort und bedeckt dasselbe auf eine kurze Strecke. An mehreren Stellen wird ein Syncytium dadurch vorgetäuscht, daß Zottenquerschnitte die Oberfläche des Fibrins berühren. Die Fibrinschicht ist von gleichmäßiger, im ganzen geringer Dicke. Sie scheint zumeist direkt der Oberfläche des Chorion aufzusitzen, nur an wenigen Stellen ist die Zellschicht unter ihr deutlich sichtbar. Zuweilen ist diese letztere einschichtig, an anderen Stellen zählt sie 2--3 Lagen von Zellen. Da wo sie nur aus einer Zelllage besteht, ist jede Zelle für sich von kompaktem Fibrin eingeschlossen, und zwischen Zelle und Fibrin ein Zwischenraum vorhanden; wo die Zellen zu mehreren übereinauder liegen, sind sie oval, meist polyedrisch, eher klein und liegen häufig dem Chorion parallel. Selten finden sich wirkliche Zellhaufen vor, die dann nischenartig ins Chorionbindegewebe hinein vorspringen. Viele der Zellen der Zellschicht, besonders die rings vom Fibrin eingeschlossenen, weisen Veränderungen in ihrer Struktur auf, zeigen ein gekörntes Protoplasma, der Kern verliert seine Färbbarkeit und hebt sich zuweilen noch als Schatten vom Zelleib ab. Der Zellleib verliert seine scharfe Begrenzung and zerfällt in kleine Schollen. Direkt auf dem Chorion treten diese Protoplasmaschollen stellenweise untereinander in Berührung, so daB zwischen Chorion und kompaktem Fibrin sich eine aus kleinen Schollen bestehende Zone einschiebt. Auf den Strecken, auf denen das Fibrin direkt dem Chorionbindegewebe anfzusitzen scheint, ist die Grenze gegen das Chorion weniger scharf da, als da wo die Zellschicht an das Chorion stößt. Das Fibrin besteht meistens aus kompakten Balken, weist zahlreiche Kanäle auf, die in der Hauptsache dem Chorion parallel verlaufen, untereinander durch schräge oder senkrechte Äste in Verbindung stehen, und je weiter vom Chorion entfernt, um so unregelmäBiger und breiter werden. In diesen Kauälen sind zahlreiche Kerne sichtbar, die meist rund, oval, oft lang gestreckt, der Form der Kanäle angepaßt, vor allem sehr klein und dunkel sind. Diese Kerne, vermischt mit Kernen mehrkörniger Leukocyten, finden sich durch die ganze Fibrinschicht ziemlich gleichmäßig verteilt vor. Die kompakten Balken des Fibrins grenzen meist gegen den intervillösen Raum hin scharf $a b$, stellenweise löst sich der dem intervillösen Raum zunächst liegende Fibrinbalken in ein zuerst grob- dann feinfaseriges Fibrinnetz auf, das feiner wird und allmählich unter Einschließen von roten und weißen Blutkörpern in den Inhalt des intervillösen Raumes übergeht.

Im Chorionbindegewebe fehlen die Kapillaren durchwegs.

$$
\text { Placenten, nahezu ausgetragen. }
$$

Placenta XI ist eine Placenta marginata und rührt von einer V. para her. 3 Wochen vor Ende der normalen Schwangerschaft trat plötzlich starker Wasserabgang auf (Hydrorhoea uteri), auf den die Geburt eintrat. Das Kind lebt. Frühere Geburten waren normal.

Die Placenta ist eine Marginata. Der Durchmesser des vom Chorion 
placentare bedeckten Teils der Placenta mißt $12 \mathrm{~cm}$, derjenige der ganzen Placenta beträgt 14,5 cm. An der Stelle der zentral gelegenen Nabelschnurinsertion hat die Placenta eine Dicke von $3,6 \mathrm{~cm}$, am Rande von $2,5 \mathrm{~cm}$. Die Dicke des Chorion samt dem aufgelagerten Fibrin beträgt 5-7 mm, an einer Stelle $12 \mathrm{~mm}$.

Das Syncytium ist am Chorion nur an wenigen Stellen vorhanden und zeigt, sowie die unter ihm sich befindende einreihige Schicht der Zellen der Zellenschicht das gewöhnliche Verhalten. Der größte Teil der Oberfläche des Chorion wird von einer stellenweise bis $12 \mathrm{~mm}$ Dicke erreichenden Fibrinschicht bedeckt, die zahlreiche Zotten einschließt. Die mittleren Teile der Fibrinschicht werden von einem Gewirr von Fibrinfäden eingenommen, in dem einige Balken kleinscholligen Fibrins hervortreten. Gegen das Chorion und gegen den intervillösen Raum hin nehmen diese Faserzüge allmählich mehr einen parallelen Verlauf an, verschmelzen zu dicht gelagerten breiten kompakten Balken, die zwischen sich nur schmale verästelte Kanäle lassen. Diese Kanäle werden gegen den intervillösen Raum hin immer zahlreicher, gröber, besitzen einen oft zackigen Querschnitt; chorionwärts sind sie seltener, feiner, liegen einander parallel und haben einen runden Querschnitt. Diese feinen Kanäle weisen in ihrem Verlauf Erweiterungen auf, in denen sich dunkel gefärbte, rundliche, ovale bis längliche mit Fortsätzen versehene Kerne vorfinden, die, je mehr sie gegen den intervillösen Raum hin liegen, um so zahlreicher sind und durchschnittlich die Größe eines weißen Blutkörperchens besitzen. Meist schiebt sich zwischen Chorion und Fibrin die Zellschicht ein, die streckenweise ein- bis zweischichtig ist, streckenweise ganze Zellhaufen, die nischenartig ins Chorionbindegewebe hinein vorspringen, bildet. Zuweilen ist die Zellschicht nicht mehr zu sehen; das Fíbrin scheint hier direkt die Chorionoberfläche zu berühren. Öfters schieben sich noch plankonvexe Lücken, mit Konvexität gegen das Fibrin hin zwischen Fibrin and Chorion ein, in denen nekrotische Zellen mit dem ungefürbten Schatten eines Kernes sichtbar sind. Da wo das kompakte Fibrin an die Zellhaufen stößt, sendet es Fortsätze aus, die in Form von Fibrinbalken in die Zellhaufen eindringen, Gruppen von Zellen umschließen und sich zwischen den einzelnen Zellen in immer feinere Âste auflösen. Die Zellen besitzen einen hellen Zellleib, einen blassen, rund-ovalen Kern mit Kerngerüst und Kernkörperchen. An der oberen Grenze der Zellschicht finden sich häufig aus dem Zellverband losgelöste, ganz ins Fibrin eingesprengte Zellen vor, die häufig Zeichen des Untergangs aufweisen. Im Chorion, auf den kurzen Strecken, wo das Syncytium noch erhalten ist, sind zahlreiche Kapillaren vorhanden, meist 1-2 pro Gesichtsfeld, bisweilen 4. Einzelne zeigen eine Adventitia. Da wo das Chorion von Fibrin bedeckt ist, fehlen die Kapillaren durchwegs.

Placenta XII, nahezu ausgetragen, rührt von einer mit Tuberculosis pulmonum behafteten Frau her. Die Placenta ist ziemlich dünn. Das Chorion weist eine kontinuierliche Fibrinschicht, teils als dünne Lage, teils 
von erheblicher, der Breite des Chorion gleichkommender Dicke auf, die zahlreiche Zotten in sich birgt, und meist direkt an das Chorion zu stoßen scheint. Die Zellschicht ist nur streckenweise und in Resten vorhanden. Das Syncytium findet sich nur auf einer ganz kurzen Strecke vor und zwar bedeckt es daselbst, auf $5-6$ Gesichtsfelder Länge eine dünne kompakte Fibrinschicht, die unmittelbar, ohne Zellen der Zellschicht in sich einzuschließen, auf dem Chorion aufsitzt. In dem unter dieser Fibrinschicht sich befindenden Chorion sind keine Kapillaren wahrzunehmen. Besonders da, wo die Fibrinschicht dick ist, finden sich auf dem Chorion noch Zellen der Zellschicht vor, die teils vereinzelt ins Chorion sich einbuchten und oft ganz von Fibrin umschlossen sind, oder zu Haufen von 15-20 Zellen beisammen liegen. Die einzelnen Zellen haben einen leicht eosinrot gefärbten Zelleib, sind größer gegen die Basis der Zellhaufen hin als gegen dessen Konvexität, Zwischen die Zellen hinein dringen Fibrinfäden, die sich in lockere Bündel auflösen, und die placentarwärts in die die Zellhaufen scharf begrenzende kompakte Substanz des Fibrins übergehen. In das Fibrin sind vereinzelte aus dem Zellverband losgelöste Zellen der Zellschicht eingesprengt, die meist Zeichen des Untergang aufweisen, einen schlecht gefärbten meist kleinen, unscharf begrenzten Kern besitzen; oder der Kern ist ganz geschwunden, und nur kernlose Schollen vorhanden, die durch einen Spaltraum von dem sie einschließenden kompakten Fibrin getrennt sind. Ferner finden sich Lücken im Fibrin von der Größe einer Zelle, oder einer Gruppe von solchen, die nur kleinere homogene Schollen enthalten, welche Bruchstücke von nekrotischen Zellen zu sein scheinen. Je weiter diese Lücken vom Chorion entfernt im Fibrin liegen, um so kleiner sind sie; sie werden zugleich unregelmäBiger schmäler, zeigen Fortsätze, die feiner werden, untereinander kommunizieren und unter sich und dem Chorion parallel verlaufen. Kerne sind in ihnen nur selten sichtbar, sind schmal, länglich, unregelmäßig geformt. Zuweilen grenzt das kompakte Fibrin direkt und scharf an den intervillösen Raum an; an anderen Stellen schiebt sich noch eine Schicht balkig-kömigen und -kleinscholligen Fibrins zwischen beide ein, die feinfasriger wird und unter Einschließung von weißen und roten Blutkörpern in den Inhalt des intervillösen Raums übergeht. Auf einer Strecke findet sich noch eine zweite Art des Ubergangs vom Fibrin in den Inhalt des intervillösen Raums. Die dünne kompakte, nur vereinzelte Zellen der Zellschicht einschließende Schicht Fibrin ist an der Oberfläche gegen den intervillösen Raum hin von einer Schicht, teils abgrenzbarer, teils gegen das Chorin zu zusammengeflossener roter Blutkörper bedeckt. Die eingehende Beschreibung dieser Zone findet sich weiter hinten in der Zusammenfassung. Wie schon erwähnt, schließt das Fibrin da, wo es dick ist, zahlreiche Zotten, längs and quer im Schnitte getroffen, ein. Diese Zotten sind durchschnittlich kernarm. Thre Grenze gegen das sie einschließende stets kompakte Fibrin ist unscharf. In ihrer Umgebung sind zahlreiche vereinzelte oder zu Gruppen beisammen liegende Zellen der Zellschicht der Zotten im Fibrin einge- 
schlossen. Kapillaren sind im Chorion keine vorhanden, nur spärliche Gefäße mit kapillarem Lumen finden sich im oberen Drittel des Chorionbindegewebes vor und zwar kommen auf 96 Gesichtsfelder 6 solche Gefäße.

\section{Ausgetragene Placenten.}

Placenta XIII. Das Fibrin ist auf der ganzen Oberfärche des Chorion in ziemlich dicker Schicht vorhanden und scheint zumeist direkt das Chorion zu berühren. Der Anteil des kompakten Fibrin an der Zusammensetzung der Fibrinschicht ist ein geringer. Nur gegen das Chorion hin weist das Fibrin 1 bis 2 Balken kompakten Fibrins auf. Da wo Zellen der Zellschicht noch erhalten sind, und sich zwischen Fibrin und Chorionbindegewebe einschieben, ist die Schicht des kompakten Fibrins noch schmäler, sendet aber zuweilen zwischen die Zellen hineinreichende Fortsätze aus. Sonst ist das Fibrin kleinschollig-balkig, oft zeigt es netzförmigen Bau und ist von feinen, dem Chorion parallel gerichteten Kanälen durchzogen, die stellenweise Erweiterungen zeigen, in denen sehr spärliche, drunkle runde Kerne zu sehen sind, und die, je näher sie dem Chorion zu liegen, immer mehr zusammenrücken. Die Grenze gegen den intervillösen Raum hin ist zum Teil eine scharfe, zum Teil geht das Fibrin vermittelst eines feinen Fibrinnetzes in den Inhalt derselben über. Die Zellschicht ist nur stellenweise vorhanden, die Zellen sind vereinzelt, oder sitzen zu kleineren oder größeren Zellhaufen, die bis 6 Zellagen übereinander aufweisen können, dem Chorion auf. Die Zellen sind zum Teil gut erhalten, oft aber besonders da, wo sie vereinzelt vorkommen, zeigen sie schlechte Kernfärbung und sind zumeist durch einen Spaltraum von dem sie einschließenden Fibrin getrennt. Bisweilen trifft man im Fibrin auf gleichgroße Lüeken ohne darin enthaltene Zellen. Da wo die Zellen zu Zellhauien zusammenliegen, sind sie besser erhalten, in der Größe wechselnd und zeigen die gewöhnlichen Merkmale. Die Zellhaufen werden durch Fibrinzüge in Gruppen zerlegt, die jede für sich nischenartig ins Chorionbindegewebe hinein vorspringt, und die ihrerseits von feinsten Fibrinfüden, die die einzelnen Zellen voneinander scheiden, durchzogen werden. Da wo das Fibrin unmittelbar das Chorion zu berühren scheint, ist die Grenze zwischen beiden eine gerade. Auf einem der Schnitte ist die Abgangsstelle einer großen Zotte getroffen und hier sieht man, wie das Syncytium, das die Zotte äberzieht, sich auf eine geringe Entfernung noch auf das Fibrin am Chorion fortsetzt. Auf der Zotte selbst wechselt das Syncytium öfters mit kurzen und dünnen Fibrinschichten ab. Auf 20 Gesichtsfelder, auf denen sich auf der Zotte Fibrin zeigte, die Basis der Zotte mit eingerechnet, fallen 2 Kapillaren, auf 31 Gesichtsfelder mit Syncytium 56 Kapillaren dicht unterhalb der Zellschicht der Zotte gelegen. Auf den wenigen und kurzen Strecken, wo das Chorion ein Syncytium aufwies, im ganzen auf einer Länge von 8 Gesichtsfeldern, fanden sich 8 Kapillaren an der Chorionoberfläche. Auf den Strecken, auf denen das Chorion von einer dünnen Fibrinschicht bedeckt ist, fielen anf 20 Gesichtsfelder 3 Kapillaren, da wo die Fibrinschicht dick ist, dagegen nur 1 Kapillare auf 
28 Gesichtsfelder. Die letztgenannten Kapillaren zeigen sämtlich verdickte Wandungen, eine Adventitia und ins Lumen hinein vorspringende Endothelkerne.

PIacenta XIV ist ausgetragen; das Chorion zeigt streckenweise ein Syncytium; Fibrin und Syncytium wechseln am nämlichen Schnitte verschiedene Male miteinander ab. Der gröBte Teil der Oberfläche des Chorion wird aber doch von Fibrin bedeckt, das stellenweise durch eine mehrsehichtige Zellschicht vom Chorion getrennt ist, oder häufiger Zellen der Zellschicht vereinzelt oder zu Gruppen in sich einschließt, zum Teil aber auch direkt an das Chorion zu grenzen scheint, meist geringe aber ziemlich gleichmäßige Dicke zeigt. Die Grenze zwischen Chorion und Fibrin ist da, wo letzteres keine Zellen der Zellsehicht mehr einschlieBt und das Chorion zu berühren scheint, von geradem Verlaufe. Da wo Zellhaufen sich zwischen Chorion und Fibrin einschieben, wird die Oberfläche des Chorion eingebuchtet; die Zellhaufen springen unter Bildung von Nischen in das Chorionbindegewebe hinein vor und kehren ihre flachere Seite gegen das Fibrin. Das Syncytium zeigt da, wo es vorkommt, die bekannten länglichen dunklen Kerne, die meist dicht aneinander, zuweilen lang ausgezogen in kleinen Zwischenräumen auseinander liegen, und durch den feinen schmalen, stark lichtbrechenden Protoplasmasaum, in den sie eingebettet sind, untereinander in Verbindung stehen. Dieses letztere Verhalten des Syncytium findet sich besonders an den Stellen am Rande einer Fibrinschicht, wo die Zellschicht unter dem Syncytium mehrere Schichten aufweist und die einzelnen Zellen durch homogenes, zuweilen feine Faserung aufweisendes Fibrin voneinander getrennt sind. Die Kerne des Syncytium und der schmale Protoplasmasaum lassen sich an diesen Stellen bis in die Fibrinschicht hinein verfolgen und scheinen daselbst kompaktes Fibrin und balkig-kleinscholliges voneinander zu trennen, und zwar liegt das kompakte Fibrin chorionwärts, das andere den intervillösen Bluträumen zu.

Die mehrsehichtige Zellschicht, die sich zwischen Fibrin und Chorion einschiebt, weist meist 2 bis 4 Lagen von Zellen auf; die Zellen selbst sind meist oval, öfters auch polyedrisch, besitzen einen bläschenförmigen Kern mit Kernkörperchen und gleichmäBig verteiltem Chromatingerüst. In der Größe sind die Zellen wechselnd. Meist liegen größere und kleinere Zellen ungeordnet durcheinander. Nur in großen Zellhaufen findet sich die von Ackermann erwähnte Anordnung, nach der die kleineren Zellen an der Konvexität und zu beiden Ästen der Zellhaufen liegen, die größeren mehr im Innern derselben. Die einzelnen Zellen liegen meist dicht beisammen, oder sie sind voneinander durch schmale Fibrinbalken getrennt, die stellenweise in große Massen übergehen, welche auf der ganzen Oberfläche Fortsätze anfweisen, mit denen sie zwischen die ausstoßenden Zellen der Zellschicht hinein, vorspringen. Da wo die Zellen nahe der Fibrinschicht gelegen oder in sie eingesprengt sind, zeigen sie zumeist degenerative Veränderungen. Der Kern weist grobe Chromatinkörner auf, das Kern- 
körperchen ist nicht mehr sichtbar, die Färbbarkeit des Kerns nimmt ab, er schrumpit zu einem unregelmäßigen Gebilde zusammen; der Zelleib ist gekörnt, kleiner geworden und geht stellenweise ohne scharfe Grenze in das die Zelle umgebende Fibrin über. In vielen Fällen verliert der Kern seine Färbbarkeit ganz.

Das Fibrin ist dem Chorion zunächst kompakt und sendet da, wo unter ihm sich noch eine Zellschicht vorfindet, Fortsätze in Form von Balken und Fasern zwischen die Zellen derselben, die die Zellen allseitig einschließen. Die so eingeschlossenen Zellen sind zum Teil gut erhalten, zum Teil bilden sie nur noch kernlose Protoplasmaschollen; oder es ist nur noch ein vom Protoplasma entblößter Kern zu sehen, der länglich und unregelmäßig ist und sich der Form der im Fibrin vorhandenen Lücken und Kanäle anpaßt. Gegen den intervillösen Raum hin wird das Fibrin zum Teil balkig-kleinschollig, zum Teil lamellös, ist nur von spärlichen Kanälen durchzogen, in denen spärliche, runde, oft unregelmäßige dunkle Kerne vorkommen. An der Oberfläche schließt es stellenweise Leukocyten und rote Blutkörper in sich ein und geht so, sich allmählich in ein Netz von Fibrinfäden auflösend, in den Inhalt des intervillösen Raumes über. Da wo Zottenquerschnitte nahe dem Chorion liegen, weist die Fibrinschicht des Ietzteren häufig ein Syncytium auf, und im Chorionbindegewebe direkt unter dieser Stelle finden sich vereinzelte Kapillaren vor.

Da bei dieser Placenta das Syncytium in Form von kleinen Inseln inmitten der von Fibrin bedeckten Oberfläehe des Chorion erhalten war, und die meisten der $2-3 \mathrm{~cm}$ langen Schnitte abwechselnd Fibrin und Syncytium aufwiesen, eignete sich diese Placenta besonders gut für die Beobachtung der Veränderungen, denen das Kapillarnetz des Chorion unterliegt. Es fand sich num, daß da wo ein Syncytium vorhanden war, zahlreiche Kapillaren im Chorion vorkamen, bei den einen der 8-9 genau durchgezählten Schnitten traf es 2-3 bei anderen 3-4 Kapillaren auf ein Gesichtsfeld (auf 5 Gesichtsfelder 18 Kapillaren, oder auf 55 Gesichtsfelder 130 Kapillaren). Die Kapillaren zeigen zum Teil eine Adventitia und ins Lumen hinein vorspringende Endothelkerne. Auf dem gröBten Teil der Oberfläche fand sich Fibrin vor, hier waren die Kapillaren spärlich. Da wo die Fibrinschicht dünn war, kam auf 3-4 Gesichtsfelder im günstigsten Falle 1 Kapillare, da wo sie dick war, traf es auf 10 ja 20 Gesichtsfelder erst 1 Kapillare, die zudem stets verdickte Wandungen zeigte. Auf 88 aufeinanderfolgende Gesichtsfelder kamen nur 4 Kapillaren. Da wo die Fibrinschicht dünn war, und sich Kapillaren vorfanden, waren meist auch gut erhaltene Zellen der Zellschicht im Fibrin eingeschlossen.

Die Kapillaren des Chorionbindegewebes finden sich sämtlich ganz an der Oberfläche derselben, direkt unter der Zellschicht und zwar mit Vorliebe in den Zwischenräumen zwischen je 2 ins Chorion hinein vorspringenden Zellen der 
Zellschicht, da wo das Syncytium oft das Chorionbindegewebe zu berühren scheint. Meist schieben sich 2 bis 3 Lagen faserigen Bindegewebes zwisehen Kapillare und Zellschicht ein. Der Durchmesser der Kapillaren kommt demjenigen von 2 bis 3 roten Blutkörpern gleich. Gefäße mit kapillarem Lumen und dentlicher Adventitia liegen etwas tiefer im oberen Drittel des Chorion, die größßeren Gefäße sind in der Mitte des Chorionbindegewebes. Elastische Fasern lassen sich in keinem der Gefäße nachweisen.

Wenn wir das, was den Kapillarreichtum des Chorion villosum anbetrifft, bei den angeführten in den versehiedenen Monaten untersuchten Placenten nochmals überblicken, so erhalten wir folgendes Resultat:

Bei der Placenta aus dem 3. Monat und der Mehrzahl derjenigen ans dem 4. Monat ist die Chorionoberfläche in fast ganzer Ausdehnung mit einem syncytialen Uberzug versehen, und das Bindegewebe des Chorion weist zahlreiche Kapillaren (bei 1 Kapillare pro Gesichtsfeld) auf, deren Verteilung meist ziemlich regelmäßig ist, oft aber Schwankungen unterliegt, so daß zuweilen mehrere Kapillaren in einem Gesichtsfeld vorkommen und anstoßende Gesichtsfelder wiederum der Kapillaren ganz entbehren. Die Fibrinbildung ist bei diesen Placenten noch spärlich. Bei der in letzter Linie angeführten Placenta aus dem 4. Monate (Placenta IV) ändert sich dieses Verhalten. Das Syncytium bekleidet nur den kleineren Teil der Oberfläche des Chorion villosum, während der größte Teil von Fibrin bedeckt ist. Hier sehen wir, daß da, wo eine Fibrinschicht das Chorion bedeckt, die Kapillaren äußerst spärlich sind und auf langen Strecken fehlen. Auf 95 aufeinanderfolgenden Gesichtsfeldern fand sich keine einzige Kapillare, währenddem auf den Strecken, auf denen ein Syncytium vorkommt, gewöhnlich 1 bis 2 Kapillaren in einem Gesichtsfeld sichtbar sind. Bei der aus dem 5. Monat stammenden Placenta (Placenta V) ist das Chorion fast durchweg von Fibrin bedeckt. Das Syncytium fehlt und ebenso auch die Kapillaren. Bei jeder der drei aus dem 6. Monat stammenden Placenten sieht man auf den kurzen Strecken, auf denen ein Syncytium vorkommt, Kapillaren an der Oberfläche des Chorionbindegewebes, die bei Placenta VI 
und VIII spärlich sind, auf 4 bis 5 Gesichtsfelder kommt nur 1 Kapillare, bei VII weniger spärlich, aber unregelmäßig verteilt sind, so daß zuweilen nur 1 Kapillare, dann wieder 4 bis 5 Kapillaren in einem Gesichtsfelde vorkommen; daneben gibt es wieder 2 bis 3 Gesichtsfelder, in denen keine Kapillaren zu sehen sind. Da wo die Fibrinschicht noch geringe Dicke besitzt, kommen noch ganz vereinzelte Kapillaren vor, da wo sie dicker wird, fehlen sie ganz. Aus dem 8. Monat haben wir 2 Placenten (IX und X), bei der einen (Placenta IX) ist das Syncytium nur auf kurze Strecken vorhanden; der Kapillarreichtum des Chorionbindegewebes an diesen Stellen ist groß, auf ein Gesichtsfeld fallen durchschnittlich 2 bis 3 Kapillaren. Da wo eine Fibrinschicht an Stelle des Syncytium das Chorion bedeckt, sind die Kapillaren sehr selten. 1 Kapillare auf 10 bis 12 Gesichtsfelder. Auf den Strecken, auf denen das Syncytium eine mehrschichtig angeordnete und zwischen den einzelnen Zellen Fibrin aufweisende Zellschicht bedeckt, nimmt die Zahl der Kapillaren an der Chorionoberfläche einen mittlern Wert an. Es kommt hier 1 Kapillare auf 1 bis 3 Gesichtsfelder.

Bei der zweiten Placenta aus dem 8. Monat (Placenta X) fehlt das Syncytium ganz und mit ihm fehlen die Kapillaren an der Oberfläche des Chorion.

Die beiden nahezu reifen Placenten (XI and XII) zeigen dasselbe Verhalten. Auf den wenigen und kurzen Strecken, an denen bei Placenta XI ein Syncytium vorkommt, finden sich Kapillaren am Chorion oft bis 4 in 1 Gesichtsfeld.

Auf dem überwiegend größten Teil der Chorionoberfläche liegt eine Fibrinschicht und hier fehlen Kapillaren durchweg. Bei Placenta XII ist das Chorion villosum in ganzer Ausdehnung mit einer Fibrinschicht versehen, Kapillaren fehlen hier ganz. Bei den beiden reifen Placenten sind die Gefäßverhältnisse folgende: Bei Placenta XIII finden sich an den wenig ausgedehnten Stellen, auf denen ein Syncytium vorkommt (8 Gesichtsfelder im ganzen), Kapillaren vor, 1 Kapillare pro Gesichtsfeld. Da wo die Fibrinschicht dünn ist, sind die Kapillaren sehr spärlich (1 Kapillare auf 6 Gesichtsfelder) und zeigen alle verdickte Wandungen. Da wo das Fibrin dick ist, 
findet sich nur selten eine ebenfalls verdickte Kapillare vor, 1 Kapillare auf 28 Gesichtsfelder. Bei der letzten Placenta (XIV), von der 8-9 Schnitte genau durchgezählt wurden, fanden sich überall da, wo Fibrinbildung am Chorion vorkam, die Kapillaren im Chorionbindewebe sehr spärlich. In den günstigsten Verhältnissen traf es bei dünner Fibrinlage $1 \mathrm{Ka}$ pillare pro Gesichtsfeld, meist war aber das gegenseitige Verhältnis ein anderes. Auf 4 bis 10 Gesichtsfelder kam $1 \mathrm{Ka}$ pillare, bei wieder anderen Schnitten traf es auf 20 Gesichtsfelder nur eine und dazu verdickte Kapillare. Da wo an Stelle der Fibrinsehicht ein Syncytium vorkam, war der Kapillarreichtum durchweg ein großer. Pro Gesichtsfeld fanden sich 3 bis 4 Kapillaren vor.

Sehr beweisend sind auch die Stellen des Chorion villosum, an denen die Abgangsstelle eines Zottenstammes vom Schnitte getroffen ist. Die Zotte durchbricht die dem Chorion aufliegende Fibrinschicht, ein schmaler Fibrinstreifen setzt sich auf die Zotte fort und läßt sich noch auf 1 bis 10 Gesichtsfelder auf der Zotte verfolgen. An seine Stelle tritt dann das die Zotten bekleidende Syncytium. Solche Stellen finden sich öfters bei den verschiedenen Placenten vor. Auf den vom Zotten-Syncytium bekleideten Strecken finden sich zahlreiche normale Kapillaren vor, bei Placenta XWI fanden sich durchschnittlich 2 Kapillaren pro Gesichtsfeld. An den Stellen, an denen der Fibrinstreifen die Zotte bedeckt, fehlen die Kapillaren ganz oder sind nur sehr spärlich (bei Placenta XIII 1 Kapillare auf 10 Gesichtsfelder) und zeigen verdickte Wandung.

Gelegentlich ist die Fibrinschicht am Chorion villosum auf eine kurze Strecke von Syncytium unterbrochen, welches der Zellschicht aufliegt. An der Mehrzahl dieser Stellen finden sich an der Chorionoberfläche vereinzelte, meist verdickte Wandung aufweisende Kapillaren vor, währenddem nach beiden Seiten hin unter der anstoßenden Fibrinschicht die Kapillaren auf langen Strecken ganz fehlen,

Wir können also das Resultat kurz zusammenfassen in dem Satz:

"Da wo ein Syneytium das Chorion villosum bekleidet, zeigt die Chorionoberfläche zahlreiche Ka- 
pillaren; $d a$ wo es fehlt und an seiner Stelle eine Fibrinschicht auf dem Chorion liegt, werden die Kapillaren sehr spärlich, ja fehlen auf langen strecken vollständig.

Es sprechen also meine Untersuchungen durchaus zugunsten der von Langhans ausgesprochenen Idee, daß die Existenz des Syneytium an diejenige eines Kapillarnetzes im gegenüberliegenden foetalen Bindegewebe gebunden ist. Beide schwinden in der $z$ weiten $H$ älfte der Schwangerschaft fast vollständig und das Syncytium wird durch Fibrin ersetzt. Syncytium und foetale Kapillaren gehoren also in funktioneller Beziehung zusammen und von denen das fötale Bindegewebe bedeckenden epithelialen Schichten kommt jedenfalls dem Syncytium der Hauptanteil an dem Stoffaustausch zwischen Mutter und Frucht zu, wie dies ebenfalls Langhans zuerst ausgesprochen hat.

Ich habe noch einige wenige Bemerkungen über die Struktur der Kapillaren anzufügen.

In den Placenten der frühern Monate, besonders da, wo das Syncytium noch größere Teile der Chorionoberfläche bekleidet, zeigt das Chorion villosum normale Kapillaren. In den spätern Monaten, wo die vom Syncytium bekleideten Strecken wenig ausgedehnt sind, zeigen sie vielfach eine Verdickung ihrer Wandung, die einesteils dadurch zustande kommt, daß sich eine fasrige Adventitia gebildet hat, die an Dicke dem halben Durchmesser des Lumens gleichkommt, und einige, 1 bis 2 längliche konzentrisch gelagerte Kerne enthält. Andrerseits, und dies ist das Häufigere, schwellen die platten Endothelien an, der lange sehmale Kern wird kugelig und springt stark ins Lamen hinein vor, so daß letzteres unregelmäßig eingeengt eine schmale wellenförmig verlaufende Linie bildet. Das Lumen kann ganz geschwunden sein. Man sieht dann beim Querschnitt einen rundlichen Haufen von Kernen, von denen die äußern länglich gestaltet und konzentrisch angeordet sind; die innersten zwei bis drei Kerne dagegen rundlich erscheinen. Diese lassen sich beim Schrauben der. Mikrometerschraube in die Tiefe verfolgen. Die äußern, konzentrisch gelagerten Kerne bilden 3 bis 4 Lagen. 
Schon Langhans hat in seiner ersten großen Arbeit die Veränderungen der Endothelien, das Anschwellen der Kerne derselben beschrieben. Auf der Außenfläche der Wandung der Kapillaren fand er in einer homogenen Grundsubstanz zahlreiche Zellen, spindelförmige und runde, mit viel Protoplasma, aber relativ kleinem Kern. Diese Zellen, die dicht beisammen liegen, bilden eine dicke Adventitia, die die Gefäße von allen Seiten umgibt. Zuweilen gelang es $\mathrm{ihm}$, im Zentrum noch ein Lumen nachzuweisen, oft fehlte ein solches. Diese Zellen der Adventitia sollten nach seiner damaligen Ansicht wuchern, in solide Zellstränge sich umwandeln, und so eine durch ihren Zellreichtum ausgezeichnete Schicht bilden, von der aus die spätere Zellschicht entstünde. In seiner späteren Arbeit über die Zellschicht hat Langhans bekanntlich die Zellschicht als eine kontinuierliche, unter dem Syncytium liegende Schicht von den frïhesten Stadien nachgewiesen und damit die Unrichtigkeit der oben erwähnten Genese festgestellt.

Wir haben im vorhergehenden die Abbängigkeit von Syncytium and foetalen Kapillaren nachgewiesen und können uns fragen, welches der beiden zuerst schwindet. Ist es das Syncytium und ist der Schwund der Kapillaren ein sekundärer oder ist es umgekehrt? Die vorliegenden Untersuchungen erlauben nicht diese Frage mit Sicherheit $\mathrm{zu}$ entscheiden. Einerseits fanden sich bei jeder Placenta Stellen, wo das Chorion, das heißt die auf demselben liegende Fibrinschicht, auf eine kurze Strecke ( 1 bis 2 Gesichtsfelder Länge) ein Syncytium aufwies, das Chorionbindegewebe aber häufig keine Kapillaren besaß. Es ist dabei allerdings zu achten, daß durch der Fibrinschicht anliegende Zottendurchschnitte ein Syncytium auf dem Fibrin vorgetäuscht werden kann. - Andrerseits, und dies ist weitaus das Häufigere, kamen auch unter der Fibrinschicht vereinzelte, wenn auch verdickte Wandung aufweisende Kapillaren vor, ohne daß ein Syncytium an der Oberfläche der Fibrinschicht oder in derselben hätte nachgewiesen werden können. Der Umstand, daß da, wo die Fibrinschicht dünn ist und die Zellschicht noch erhalten ist, mehr Kapillaren am Chorion sich vorfinden, als da, wo das Fibrin dick und die Zellschicht untergegangen ist, ließe den primären Schwund des Syncytium 
wahrscheinlicher erscheinen. Dies würde auch mit den Anschauungen von Langhans übereinstimmen; nach demselben liegt dem Syncytium die Aufgabe ob, den Stoffanstausch zwischen Mutter und Frucht zu ermöglichen. Man könnte so annehmen, daß infolge der größeren Entfernung der Chorionoberfläche von der Serotina, wie in der Einleitung auseinandergesetzt, das Syncytium zuerst in seiner Funktion leidet und erst später die Kapillaren zugrunde gehen, weil sie von dem Syncytium her nicht mehr die notwendigen Stoffe erhalten.

In den Beschreibungen der histologisehen Bilder der einzelnen Placenten habe ich des öftern betont, daß die unter dem Syncytium einreihig vorhandene Zellschicht am Rande einer Fibrinschicht mehrsehichtig wird und oft in 2 bis 4 , ja zuweilen in 10 bis 15 Lagen sich zwischen Fibrin und Chorion einschiebt. Ist diese Wucherung der Zellschicht mit dem Schwunde des Syncytium in Einklang zu bringen? Ist sie die Folge desselben? Das regelmäßige, sozusagen gesetzmäßige Vorkommen dieses Verhaitens scheint dafür zu sprechen.

Wie ich eingehends anführte, habe ich auch des genaueren auf die Beschaffenheit des Syncytium, der Zellschicht und des Fibrin am Chorion bei den von mir untersuchten Placenten geachtet.

Das Syncytium besteht aus nebeneinander, mehr oder weniger dicht gelagerten Kernen, die dunkel gefärbt sind, grobe Chromatinkörner gleichmäßig im Innern des Kernes verteilt, aufweisen, meist der Oberfläche des Chorion parallel liegen und in einen gemeinsamen, schmalen gekörnten Protoplasmasaum und zwar in dessen tieferen Schichten, eingebettet sind. Thre Länge ist Schwankungen unterworfen; oft übertrifft sie die Breite nur um weniges, bisweilen um das drei- bis vierfache. Am Rande einer Fibrinsehicht schlägt sich das Syncytium auf diese über, weist anfänglich noch Kerne auf und geht dann, nachdem es sich eine kurze Strecke als lichtbrechender Protoplasmasaum zwischen die Lagen des dicker gewordenen Fibrin verfolgen läßt, in die Fibrinbalken über. Stellenweise ist das Syncytium am Rande einer Fibrinschicht auf größere Strecken unter einer dicken Fibrinlage sichtbar und liegt dabei auf einer einreihigen normalen Zellschicht. Dies 
ist der Fall, wenn die Fibrinplatte pilzförmig das anstoßende, von einem Syncytium bedeckte Chorion überragt. An anderen Stellen wiederum ist es in Form von Zellhaufen zwischen Fibrin und zwischen Zellschicht zusammengepreßt.

Die Zellschicht ist teils einschichtig und dann von einem Syncytium bedeckt, oder mehrschichtig and zeigt dann meist eine sie überziehende Fibrinschicht. Stellenweise ist sie ganz geschwunden und das Fibrin scheint die Oberfläche des Chorion zu berühren. Die Grenze gegen das Chorion ist an diesen Stellen oft unscharf. Da wo die Zellschicht einschichtig ist, besteht sie aus annähernd kubischen und ovalen Zellen, die eine jede ins Chorionbindegewebe hinein vorspringt, mehr oder weniger dicht gelagert sind, zuweilen Zwischenräume, in denen das Syncytium die Oberfläche des Chorion zu berühren scheint, zwischen sich lassen. Der Kern ist bläschenförmig, meist oval, blaß, enthält ein meist gleichmäßig verteiltes feines Chromatingerüst und ein oder mehrere Kernkörperchen. Da wo die Zellschicht mehrschichtig ist, bildet sie eine kontinuierliche Lage, die meist 3-5 und mehr Schichten zählt, oder sie setzt sich aus einzelnen Zellhaufen zusammen, in denen oft nur wenige Zellen, bisweilen aber bis zu 15 und mehr übereinander liegen. Diese Zellhaufen wölben sich nischenartig ins Chorionbindegewebe hinein vor. Gegen das Fibrin hin sind sie abgeflacht, selten zeigen sie auch gegen dieses hin eine konvexe Fläche. Die Größe der Zellen wechselt. Häufig sind sie kaum größer als ein weißes Blutkörperchen. Im allgemeinen liegen die kleineren Elemente mehr chorionwärts; bei reifen Placenten findet sich in großen Zellhaufen das von Ackermann erwähnte Verhalten, nach dem die großen Zellen der Mitte und der Basis derselben zu liegen. Meist gehen Größe der Zelle und Größe des Kerns einander pärallel. Die einzelnen Zellen platten sich gegenseitig ab, die polygonale Form der Zellen überwiegt infolgedessen. Die Zellgrenze ist als eine feine lichtbrechende Linie sichtbar, meist aber, besonders in den Zellhaufen, wodurch wohl deren Vorwölbung gegen das Chorionbindegewebe zustande kommt, schieben sich verschieden dicke Züge und Balken einer homogenen Substanz, oft Komplexe von Zellgröße, zwischen die einzelnen Zellen ein. Auch da wo die Zellschicht 
mehr eine gleichmäßige Breite hat, sieht man in ihrem Innern Fasern und Balken, deren gröbere dem Chorion parallel verlaufen, wodurch ein etagenähnlicher Aufbau zustande kommt. Diese Fasern und Balken, die mit feinsten Zügen die einzelnen Zellen umspinnen, gehen an der oberen Grenze der Zellschicht in einen dicken Balken kompakten Fibrins über; mit dem sie das lichtbrechende Vermögen teilen. Eberhardt hat nachgewiesen, daß diese scheinbare Intercellularsubstanz deutliche Fibrinreaktion zeigt, bei Färbung mit Pikrinsäure (Methode nach Neumann) und bei Färbung nach Weigert. Ackermann bestätigte dies Resultat und ich habe mich auch von der Richtigkeit desselben, sowohl an Präparaten, die der Eberhardtschen Arbeit zugrunde liegen, als an eigenen überzeugt. - Die Zellhaufen schneiden meist, zumal in den ersten Monaten, in gerader Ebene gegen das sie bedeckende Fibrin ab. Vom 8. Monat ab findet man aber meist aus dem Zellverband losğelöste Zellen der Zellschicht mitten in das kompakte Fibrin eingesprengt, wie auch die die obersten Lagen der Zellhaufen bildenden Zellen von dicken Fibrinbalken allseitig umschlossen werden. Die so eingeschlossenen Zellen füllen den ihnen im Fibrin eingeräumten Raum oft nicht ganz aus, zwischen Zelle und Fibrin besteht ein Zwischenraum. Die Zellen selbst zeigen Merkmale ihres Untergangs, die, je näher man sie nach oben gegen das kompakte Fibrin hin verfolgt, um so ausgesprochener sind. Das Protoplasma der Zellen wird gekörnt, der Zelleib kleiner, der Kern oft unregelmäßig, zackig, die Chromatinkörner liegen der Kernmembran an, während das Innere aufgehellt ist, oder sie sind zu größeren Klumpen zusammengeballt. Der Kern verliert seine Färbbarkeit, tritt nur noch als ein Schatten in dem zu kleinen Schollen zerfallenen Zelleib hervor. Die Spalträume, die sich meistens zwischen den nekrotisierenden Zellen und dem diese einschließenden Fibrin bilden, treten gegen die Fibrinschicht zu untereinander in Verbindung und bilden ein unregelmäßig gestaltetes, an Breite wechselndes System von Kanälen, die rhombische Fibrinkomplexe begrenzen (globuläre Anordnung), in dem aber bald dem Chorion parallel verlaufende Kanäle überwiegen. Diese letzteren werden nach oben hin feiner, gleichmäßig schmal, liegen dicht übereinander, 
kommunizieren durch kurze, senkrecht oder schräg verlaufende Äste miteinander und bilden die kompakte Form des kanalisierten Fibrins. Zuweilen besteht die ganze Fibrinschicht nur aus kompaktem Fibrin, meist geht aber letzteres früher oder später, oft schon nach wenigen Balken in kleinschollig-balkiges oder in lamellös-retikuläres Fibrin über. Die Kanäle werden hier wieder unregelmäßig in ihrer Breite und in ihrem Verlauf. Gegen den intervillösen Raum hin schneidet das Fibrin häufig scharf $a b$, oder es geht in ein einer diphtheritischen Membran ähnlich sehendes Netz von Fibrinfäden über, das feiner und feiner wird und unter Einschließen von Leukocyten, Lymphocyten und roten Blutkörperchen allmählich in den Inhalt des intervillösen Raumes ausmündet. Bisweilen ist aber der Ubergang in den Inhalt des intervillösen Raumes ein anderer (vide Placenta XII). Eine gelbliche Zone von meist geringer Breite schiebt sich zwischen Fibrin und dem aus roten und weisen Blutkörperchen bestehenden Inhalt des intervillösen Raumes ein. Es sind rote Blutkörperchen oder Teile solcher, leicht glänzend, homogen, gelbrot, also noch hämoglobinhaltig, scharf begrenzt, lichtbrechend, die zu einem feinmaschigen Netz von schmalen Balken zusammenfließen und direkt in das Fibrin äbergehen. Das Fibrin weist in den obersten Schichten, wie diese Cbergangszone eine grünliche Fluoreszenz auf. Langhans hat diese Art der Entstehung des Fibrin schon in seiner ersten Arbeit beschrieben. v. Recklinghausen hat dagegen angeführt, daß die Farblosigkeit des Fibrin gegen diese Genese spräche. Ich teile diese meine Beobachtung nur mit, um zu zeigen, daß die Angaben von Langhans doch nicht unbegründet waren.

In den Kanälen des kanalisierten Fibrins kommen vielfach Kerne vor, die meist gegen den intervillösen Raum hin zahlreicher sind, meist dem Verlauf der Kanäle sich anpassen, stäbchenförmig ausgezogen, oft buchtig mit Ausläufern versehen sind, was die Beurteilung ihres Volumens erschwert. Seltener liegen die Kerne an den Knotenpunkten der Kanäle. Diese Kerne färben sich alle dunkel mit Hämalaun, öfters finden sich anch Kerne mehrkerniger Leukocyten vor. Gegen das Chorion hin sind sie spärlicher, oft oval und weniger intensiv 
gefärbt, enthalten bisweilen noch ein Kernkörperchen und lassen sich trotz ihres kleineren Volumens von Kernen der Zellen der Zellschicht ableiten. Langhans fand (siehe seine erste Arbeit), daß die Kerne in den früheren Stadien viel dichter liegen, als in den spätern, daß also die durch sie abgegrenzten Bezirke der Grundsubstanz später größer sind als früher und zweitens, daß die Kerne in den spätesten Stadien in ziemlich großen, regelmäßigen Abständen liegen, in den jüngeren dagegen viel dichter und unregelmäßig verteilt sind. Nach ihm ließe sich dies Verhalten am einfachsten durch die Annahme eines selbständigen Wachstums des Fibrins, durch Intussuszeption, erklären.

Wir haben in der Einleitung gesehen, daß Langhans das Fibrin teils von der Zellschicht (kompakte Form), und teils vom mütterlichen Blute (lamellöse Form) her ableitete. Ackermann kam in seinen Untersuchungen zu anderen Ergebnissen. Nach ihm soll die lamellöse Form genetisch identisch mit der kompakten Form sein, beide von der. Zellschicht des Chorion abstammen. Die Zellen der Zellschicht sollen ein Sekret liefern, das zunächst homogen ist (kompaktes Fibrin), dann bald eine erst spärliche, dann allmählich immer dichtere fibrilläre Zerklüftung zeigt und schließlich in eine zahllose Menge feinster, dicht verfilzter Fasern übergeht (lamellöses Fibrin). Die Zellen selbst sollen in kleinere und größere Schollen zerfallen, die sich zwischen den. Lamellen anhäufen. Sie beteiligen sich also nicht direkt am Aufbau der Grundsubstanz des kanalisierten Fibrins.

Ich glaube, die oben von mir beschriebenen Übergangsbilder lassen die Entstehung des Fibrins vom mütterlichen Blute aus außer jeden Zweifel stellen. Die Ansicht von Ackermann, nach der das Fibrin eine Art Sekret der Zellen der Zellschicht sei, ist ja verlockend. Einen ähnlichen Vorgang hat v. Recklinghausen für die Entstehung des Hyalins beschrieben. Das, was Hyalin wird, soll sich aus dem Zellprotoplasma infolge von Wasserverlust oder infolge von Druckwirkungen ausscheiden, häufig in Form von Kugeln, unter Zugrundegehen der übrigen Baustoffe der Zellen, der Körner des Protoplasmas und des Kernes. Doch dabei handelt es sich 
um die Genese des Hyalin und wie schon erwähnt, besitzt die scheinbare Intercellularsubstanz, die sich zwischen den Zellen der Zellschicht anhäuft und diese oft allseitig umschließt, die Charaktere des Fibrins, insbesondere auch dessen spezifische Färbbarkeit. Wir wissen, daß Fibrin nur vom Blute ans entstehen kann. Man könnte sich das Entstehen der Fibrinschicht am Chorion folgendermaßen vorstellen: Nach dem Schwund des Syncytium, das, wie wir wissen, einen gerinnungshemmenden Einfluß besitzt, bespült das mütterliche Blut die Zellen der Zellschicht. Das Blutplasma dringt nach und nach zwischen die Zellen ein; überall da wo das Blut die Zellen berührt, tritt, vielleicht infolge eines in denselben vorkommenden Enzyms, das die Gerinnung begünstigt, Gerinnung ein. Die nun vom Fibrin umschlossenen, vom Bhtplasma durchströmten Zellen sterben ab; sie fallen der Weigertschen Coagulationsnekrose anheim. Die Zellen verlieren den Kern, zeigen ein körniges oder scholliges Aussehen und gehen unter Zerfall zugrunde.

Daß sich gelegentlich bei noch vorhandenem Syncytium Fibrin unter demselben zwischen den Zellen der stets mehrschichtigen Zellschicht vorfindet, spricht nicht gegen diese Auffassung. Denn diese Stellen sind stets am Rande einer Fibrinschicht, das Plasma des Blutes kann von dort her zu ihnen vordringen.

Wie Eberhardt und Ackermann nachgewiesen haben, färbt sich das Fibrin am Chorion nach Anwendung der Weigertschen Methode; jedoch in verschiedener Stärke. Eine intensive blaue Färbung tritt konstant in den am meisten chorionwärts gerichteten Abschnitten ein, besonders intensiv an dem die Zellen der Zellschicht einschließenden Fibrin. Außerdem tritt sie regelmäßig auch in den an die intervillösen Räume anstoßenden Fibrinlagen ein. In der Mitte der Fibrinschicht färbt sich das Fibrin am wenigsten, bleibt oft ungefärbt, so daß Eberhardt an eine hyaline Umwandlung desselben dachte. Da die Resultate nicht ganz konstant waren, behielt Eberhardt die ältere Bezeichnung „Fibrin“ bei. Immerhin schien hervorzugehen, daß die Färbefähigkeit den jüngsten Abschnitten des Fibrins zukomme, das zeitlich ältere Fibrin hat die Färbefähigkeit eingebüßt. Es nimmt meist die Mitte der Fibrinschicht ein. 
Was die Genese der Kanäle im kanalisierten Fibrin anbelangt, so ließ sie Langhans aus den Kernen der Zellschicht und von weißen Blutkörperchen aus entstehen. v. Recklinghausen führt die Entstehung der Kanäle in erster Linie auf den Druck zurück, der auf die hyaline (fibrinöse) Zellreste einschließende und Vakuolen bergende Masse einwirkt. Auch Ackermann, der sich auf diese Ansicht von Recklinghausen stützt, läßt die Kanäle durch Druck und Zng, der bei der fortschreitenden Vergrößerung der Placenta entsteht, zustande kommen. Wir haben gesehen, daß die Zellen der Zellschicht, da wo sie vom Fibrin eingeschlossen werden, der Coagulationsnekrose anheimfallen. Die Zellen werden meist kleiner, zuweilen werden ihre Grenzen unscharf, sie gehen in das sie einschließende Fibrin über (Placenta XIV), häufig entsteht zwisehen ihnen und dem Fibrin ein sehmaler Spaltraum. Der Zelleib zerfällt meist in kleine Schollen, der Kern kann untergehen oder läßt sich, meist kleiner und von Protoplasma entblößt, im Fibrin noch nachweisen. Die feinen Spalten, die sich häufig zwischen Zelle und Fibrin nachweisen lassen, nehmen, je weiter man sie gegen den intervillösen Raum hin verfolgt, eine dem Chorion parallele Richtung an, sie treten untereinander in Verbindung und währenddem sie anfänglich mehr rhombische Fibrinmassen abgrenzen, gestalten sie sich nach oben zu immer mehr zu einem dichtgedrängten System unter sich und zum Chorion parallel verlaufender Kanäle, in denen noch Protoplasmaschollen und meist längliche Ausläufer aufweisende Kerne enthalten sind. Nahe dem intervillösen Raume werden die Kanäle wieder unregelmäßiger in ihrem Verlauf und meist weiter. Die dem Chorion parallele Richtung der Kanäle wird verständlich, wenn wir uns die Art und Weise des Wachstums des Chorion vorstellen. Mit der fortschreitenden Vergrößerung der Placenta wächst das Chorion in die Fläche und übt dadureh auf die ihm aufliegende Fibrinschicht, die Protoplasmakerne und häufig schmale leere Spalträume einschließt, einen konstant einwirkenden Zug aus. Kerne, Schollen und Spalträume werden in die Länge gezerrt, verursachen so zusammenhängende Kanäle, die, je länger sie diesem Znge ausgesetzt sind, das heißt, je älter das Fibrin ist, einen zur Wirkungs- 
ebene dieses Zugs, zum Chorion, parallelen Verlauf annehmen. Zugleich, da sich auch ein senkreeht auf sie einwirkender Druck, einesteils, von der Eihöhle her, andernteils der mütterliche Blutdruck geltend macht, rücken sie immer näher zusammen und sind deshalb im ältesten Abschnitte des Fibrins am dichtesten gelagert.

Zum Schlusse sei es mir gestattet, meinem hochverehrten Lehrer, Herrn Prof. Theodor Langhans, für die Anregung zu dieser Arbeit, für seine Ratschläge bei der Ausführung derselben und für die gütige Überlassung des Materials meinen herzlichsten Dank auszusprechen.

\section{Literatur.}

Th. Langhans, Untersuchungen über die menschliche Placenta. Archiv für Anatomie und Embryologie, 1877.

Derselbe, Festschrift für Jakob Henle, 1882.

v. Merttens, Beiträge zur normalen und pathologischen Anatomie der menschlichen Placenta. Zeitschrift für Geburtshilfe und Gynaekologie. Bd. XXX, 1894.

Eberhardt, Über Gerinnungen in der menschlichen Placenta. Inaug.-Diss. Bern, 1891.

Ackermann, Zur normalen und pathologischen Anatomie der Placenta. Internationale Beiträge zur wissenschaftlichen Medizin, 1891.

Nitabuch, Beiträge zur Kenntnis der menschlichen Placenta. Inaug:-Diss. Bern, 1887.

A. Kölliker, Entwicklungsgeschichte des Menschen und der höheren Tiere.

v. Recklinghausen, Allgemeine Pathologie des Kreislaufs und der Ernährung.

\section{XXVII. \\ Intraabdominelle Hernie der Bursa omentalis bei geschlossenem Foramen Winslowii.}

(Aus dem Pathologischen Institut der Universität Heidelberg.)

Von

Dr. Ernst Schwalbe,

a. o. Professor und Assistenten des Instituts.

(Mit 1 Figur im Text.)

In den folgenden Zeilen möchte ich über einen Fall innerer Hernie Mitteilung machen, der wegen seiner Seltenheit be- 